\title{
Structural, dielectric and
}

Raman spectroscopic study of complex electric and magnetic interactions in multiferroic ionic crystals

\author{
Holger Gibhardt, * Fabian Ziegler and Götz Eckold \\ Georg-August-University of Göttingen, Tammannstr. 6, D-37077 Göttingen, Germany
}

\section{Introduction}

While ordinary materials are usually either magnetically or electrically ordered, a relatively new class of materials of so-called multiferroics has been discovered that exhibits magnetic and electrical (and eventually also mechanical) ordering simultaneously. The unique and fascinating feature of these systems is that both phenomena are coupled and that electric forces may be used to control the magnetic structure and vice versa.

Unfortunately, most systems that have been investigated so far exhibit these extraordinary properties only at low temperatures. In view of possible applications in information technology, sensor technology etc., it is, therefore, a crucial task to increase the temperature range of multiferroics. Since the exact mechanism of spin coupling with the movement of ions and the resulting electric dipole moment is still not fully understood, detailed experimental and theoretical studies are needed which require advanced techniques like high-resolution

\section{DOI: 10.1255/sew.2021.a22}

(C) 2021 The Authors

Published under a Creative Commons BY licence

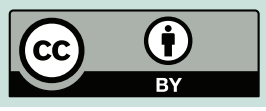

Raman spectroscopy with suitable laser sources.

One particular interesting system is $\mathrm{MnWO}_{4}$, in which the $\mathrm{Mn}^{2+}$ is the only magnetic ion. Its magnetic phase diagram is rather complex and exhibits two different antiferromagnetic phases, below $T_{\mathrm{K}}=6.8 \mathrm{~K}$ and between $T_{C}=12.3 \mathrm{~K}<T<T_{N}=13.2 \mathrm{~K}$, respectively. In the intermediate multiferroic phase between $T_{K}$ and $T_{C}$ the spins are no longer antiferromagnetically aligned but form a cycloidal wave along a certain crystallographic direction. The stabilising interactions between different spins are mediated by the diamagnetic oxygen ions by the magnetic super-exchange. Additionally, a force is exerted on the oxygen ions so that they are displaced from their normal position. In consequence, an electric dipole moment is generated and the crystal becomes ferroelectric. The electro-magnetic coupling is usually described by the "inverse Dzyaloshinskii-Moriya" interaction.

Variation of magnetic interaction is a key tool to investigate the electromagnetic behaviour of such complex systems. This can be done by doping the crystal with other ions. For example, it is possible to replace some manganese ions by non-magnetic ions which leads to a decrease of the magnetic interaction and, hence, to a reduction of the stability region of the multiferroic phase.
On the other hand, the variation of nonmagnetic ions can help to increase the ion displacements and the spin-induced electric dipole moments which lead to an enhanced multiferroic regime. In the present study, we replace tungsten by molybdenum and characterise the structural, dielectric and spectroscopic properties of the mixed system $\mathrm{MnWO}_{4}-$ $\mathrm{MnMoO}_{4}$.

Lattice vibrations reflect details of local interatomic interactions. Hence, Raman spectroscopy is a powerful tool to investigate the effect of ion doping on the spin-oxygen coupling. In a previous publication, ' we were able to demonstrate that there is an unusual strong temperature dependence of the $\mathrm{W}-\mathrm{O}$ stretching vibration within the $\mathrm{WO}_{6}$ octahedra. Using the green line of an $\mathrm{Ar}^{+}$-ion laser with a wavelength $\lambda=514.532 \mathrm{~nm}$, a shift of $0.3 \mathrm{~cm}^{-1}$ of this $884.4 \mathrm{~cm}^{-1}$ mode could be detected within the rather small temperature interval of the multiferroic phase $(5.5 \mathrm{~K})$. Variations of the magnetic interactions by Mo-doping can, therefore, be monitored by frequency shifts of this particular lattice mode.

The replacement of the tungsten ions by molybdenum ions is rather challenging and needs a multistep chemical solid-state process. ${ }^{2,3}$ After mixing the pure components, $\mathrm{MnWO}_{4}$ and $\mathrm{MnMoO}_{4}$, several cycles of ball-milling, pellet-pressing, temperature-cycling and 
final sintering were applied in order to produce well-defined samples of a series of compositions of $\mathrm{MnW}_{1-x} \mathrm{Mo}_{x} \mathrm{O}_{4}$ with $0<x<1$. Even if both pure compounds belong to the same space group $P 2 / \mathrm{m}$ and exhibit only small differences in their lattice parameters, they differ considerably in the coordination of oxygen ions. While in $\mathrm{MnWO}_{4}$ (wolframite), tungsten is six-fold coordinated by oxygen, forming $\mathrm{WO}_{6}$-octahedra, molybdenum in $\mathrm{MnMoO}_{4}$ is four-fold coordinated and $\mathrm{MoO}_{4}$-tetrahedra are the corresponding building blocks. As a consequence, there is a limited miscibility with a pronounced gap between $x=0.3$ and $x=0.65$. The exact extension is still under discussion in the literature and seems to depend on the type of preparation. The samples investigated in the present study are, therefore, characterised by X-ray powder diffraction as shown in Figure 1.

There are three concentration regimes which can clearly be distinguished:

- Group I (black): $0<x<0.3$ : the diffraction pattern consists of the reflections of the pure wolframitephase of $\mathrm{MnWO}_{4}$ (except the sample holder reflections indicated by $\mathrm{Ta}$ ). Hence, in this concentration regime, six-fold coordinated tungsten ions are gradually substituted by molybdenum ions.
- Group III (blue): $0.65<x<1$ : the diffraction pattern consists of the reflections of pure $\mathrm{MnMoO}_{4}$ and the four-fold coordinated molybdenum ions are substituted by tungsten.

- Group II (red): $0.3<x<0.65$ : the diffraction pattern resembles that of pure $\mathrm{MnWO}_{4}$ but, as indicated by an asterisk, the main reflection of the molybdate-phase is also present at $26^{\circ}$ and grows with increasing Mo-content $x$. This finding is a clear signature of the miscibility gap even if the reflections of the tungstatephase are still dominating.

\section{Raman spectroscopy}

More detailed information about the variation of interatomic interactions can be obtained by Raman spectroscopy. Unlike the previous study on pure $\mathrm{MnWO}_{4}$, however, the mixed crystalline system cannot be investigated using the standard argon laser since the molybdenum content makes the sample entirely opaque and strongly absorbs green light.

As a consequence, a green laser is inappropriate for this system and a red one must be preferred. In Raman spectroscopy, fundamental conditions of the exciting light are long-time stability as well as a narrow bandwidth of about $0.01 \mathrm{~cm}^{-1}$. Hence, we used a

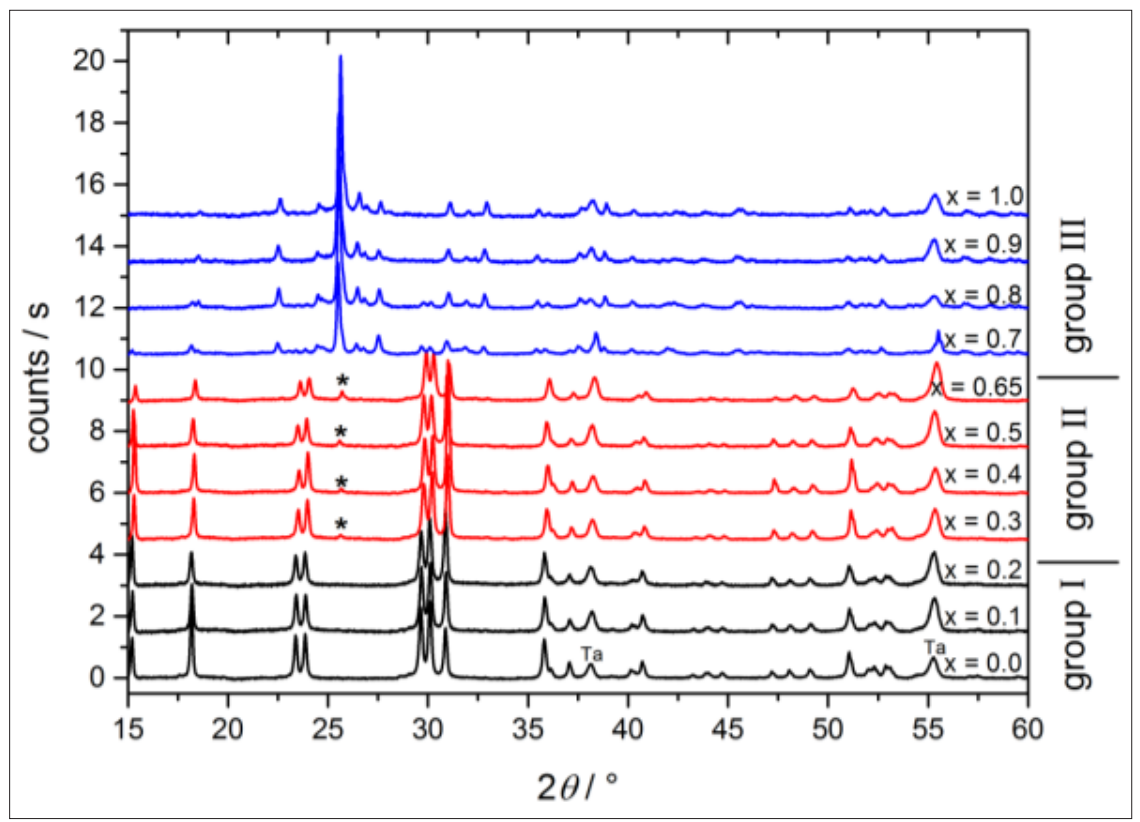

Figure 1. X-ray diffraction pattern of sintered $\mathrm{MnW}_{1-x} \mathrm{Mo}_{x} \mathrm{O}_{4}$ samples with different concentrations. Different colours represent concentration regimes with different structural characteristics. novel solid-state red laser Solo640 (UNIKLASERS), which meets these requirements very well. This laser has a wavelength of $\lambda=640 \mathrm{~nm}$ and a maximum power of $500 \mathrm{~mW}$ (at the sample, $10 \mathrm{~mW}$ are applied).

Figure 2 displays concentration dependent spectra for two ranges of wavenumbers. Again, the three concentration groups can be distinguished well as demonstrated by the colour-coded spectra.

Of particular interest is the concentration dependence of the internal stretching vibration of the oxygen-polyhedron between $850 \mathrm{~cm}^{-1}$ and $950 \mathrm{~cm}^{-1}$. With increasing Mo-content, the well-defined $\mathrm{WO}_{6}$-vibration at $884.4 \mathrm{~cm}^{-1}$ gets weaker and another mode at $850 \mathrm{~cm}^{-1}$ is observed with growing intensity which can be attributed to the molybdenum substitution at the centre of the oxygen-octahedra. In the concentration regime of the molybdate-phase (blue spectra), the Raman spectrum is split into several bands which correspond to different types of stretching vibrations in the $\mathrm{MoO}_{4}^{2-}$-tetrahedra. The external vibration at lower wavenumbers shown on the left-hand side of Figure 2 confirm the impression that up to $x=0.65$, the features of the tungstate-phase are retained with some modifications due to the Mo-doping. Only beyond $x=0.7$ the modes of the molybdate-phase dominate and the wolframite modes are strongly reduced in particular.

The effect of molybdenum doping on the multiferroic phase transition is reflected by the temperature dependence of the real part of the dielectric permittivity $\varepsilon^{\prime}$ shown in Figure 3 as obtained by dielectric impedance spectroscopy. The well-defined peak indicates the Curietemperatures $T_{C}$, where the multiferroic phase is entered on cooling associated with a spontaneous electric polarisation. With increasing molybdenum concentration, $T_{C}$ increases significantly and the multiferroic phase is stabilised at higher temperatures. The dielectric loss peak can still be observed for molybdenum concentrations up to $x=0.5$ even if the intensity decreases gradually. After an almost linear behaviour of $T_{C}(x)$ for lower concentrations just as observed by Hardy 

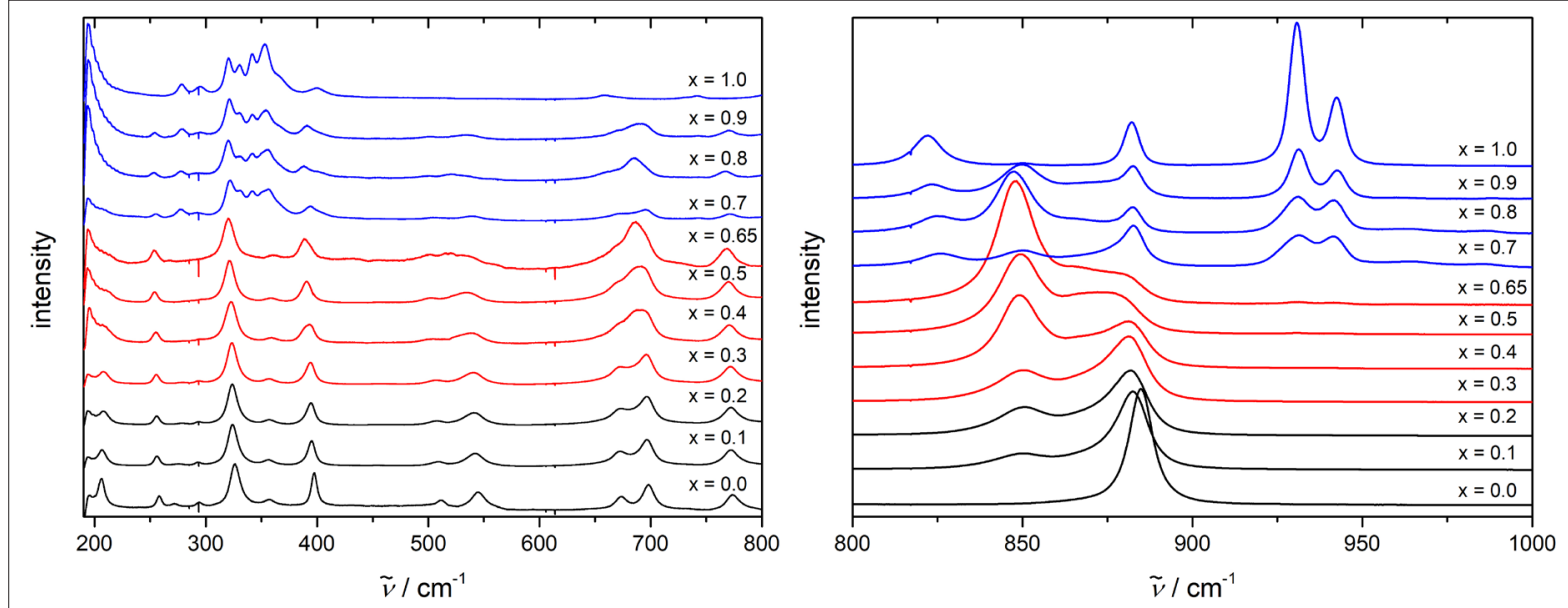

Figure 2. Raman spectra of sintered $\mathrm{MnW}_{1-x} \mathrm{Mo}_{x} \mathrm{O}_{4}$ samples with different concentrations. The three concentration regimes are distinguished by different colours. The intensities are normalised with respect to the $\mathrm{A}_{\mathrm{g}} 4$-mode at $327 \mathrm{~cm}^{-1}$.
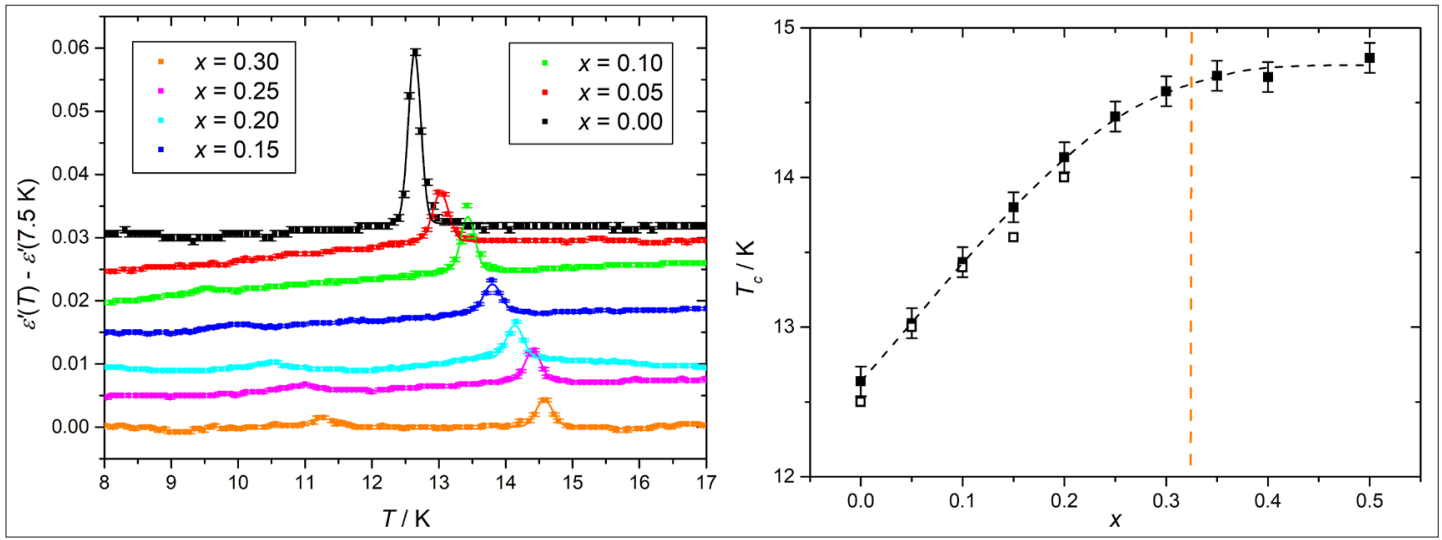

Figure 3. Left: temperature dependence of the real part of the dielectric permittivity of $\mathrm{MnW}_{1-x} \mathrm{Mo}_{x} \mathrm{O}_{4}$ for different compositions. Right: concentration dependence of $T_{\mathrm{C}}$. Open symbols are datapoints from Reference 4.

et $a l .{ }^{4}$ there is a saturation near $x=0.35$ at $T_{C}=14.7 \mathrm{~K}$ more than $2 \mathrm{~K}$ higher than for pure $\mathrm{MnWO}_{4}$.

The results of the present study indicate, in fact, that partial substitution of ions leads to an extended temperature range of the multiferroic phase. The key feature is the modification and strengthening of the super-exchange interaction between the magnetic ions mediated by oxygen. Understanding the underlying processes on the atomic level is crucial for possible applications and for the development of new materials with exciting properties.

\section{References}

1. F. Ziegler, H. Gibhardt, J. Leist and G. Eckold, "High-resolution polarised Raman scattering study on spin-phonon coupling in multiferroic $\mathrm{MnWO}_{4}$ ", Mater. Res. Express 2, 096103 (2015). https://doi.org/10.1088/20531591/2/9/096103

2. L. Meddar, M. Josse, M. Maglione, A. Guiet, C. La, P. Deniard, R. Decourt, C. Lee, C. Tian, S. Jobic, M.-H. Whangbo and C. Payen, "Increasing the phase-transition temperatures in spin-frustrated multiferroic $\mathrm{MnWO}_{4}$ by Mo doping", Chem. Mater. 24(2), 353-360 (2012). https://doi. org/10.1021/cm2031653

Dr Holger Gibhardt studied physics in Heidelberg and Aachen (Germany) and graduated from Aachen in 1994 with the "diploma". In 1998, he finished his PhD thesis at the Institute for Physical Chemistry in Göttingen. His research interests are structural and spectroscopic investigations in different condensed matter systems. Neutron scattering and Raman scattering have been the most important tools. Since, 1999, he is Senior Scientist at the Institute for Physical Chemistry in Göttingen. hgibhar@gwdg.de 